\title{
Adsorptive Granulocyte and Monocyte Apheresis Is Effective in Ulcerative Colitis Patients Both with and without Concomitant Prednisolone
}

\author{
Keiji Matsuda ${ }^{a}$ b Kohei Ohno ${ }^{a}$ Yuka Okada ${ }^{a}$ Takahiro Yagi ${ }^{a} \quad$ Mitsuo Tsukamoto ${ }^{a}$ \\ Yoshihisa Fukushima ${ }^{a}$ Atsushi Horiuchi ${ }^{a}$ Ryu Shimada, b Tsuyoshi Ozawaa, b \\ Tamuro Hayama $^{a, b}$ Takeshi Tsuchiya ${ }^{a, b}$ Junko Tamura ${ }^{a}$ Hisae linuma $^{a}$ \\ Keijiro Nozawa ${ }^{a, b}$ Hitoshi Aoyagi $^{\text {b, } c}$ Akari Isono $^{b, c}$ Koichiro Abe ${ }^{b, c}$ \\ Shinya Kodashimab, c Takatsugu Yamamotob,c ${ }^{\text {boshitaka Kawasaki }}{ }^{d}$

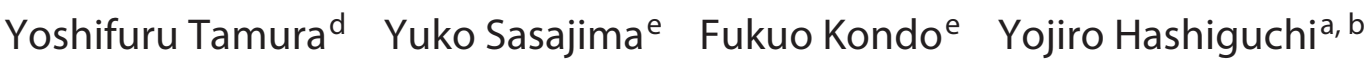

${ }^{a}$ Department of Surgery, Teikyo University School of Medicine, Tokyo, Japan; 'bIBD Center, Teikyo University School of Medicine, Tokyo, Japan; ' Department of Internal Medicine, Teikyo University School of Medicine, Tokyo, Japan; ${ }^{\mathrm{d} D e p a r t m e n t}$ of Renal Center, Teikyo University School of Medicine, Tokyo, Japan; ' Department of Pathology, Teikyo University School of Medicine, Tokyo, Japan

\section{Keywords}

Adsorptive granulocyte and monocyte apheresis .

Colonoscopy · Inflammatory bowel disease - Mayo score ·

Steroid free $\cdot$ Ulcerative colitis

\begin{abstract}
Background: The number of ulcerative colitis (UC) patients is increasing in Japan and other countries. Selective depletion of myeloid lineage leucocytes by adsorptive granulocyte and monocyte apheresis (GMA) with an Adacolumn (JIMRO, Takasaki, Japan) was introduced as a nonpharmacologic treatment strategy in UC patients in 2000. GMA has been reported to be effective in clinical trials; however, the effect of concomitant prednisolone (PSL) on GMA needs to be clarified. Methods: Thirty-nine patients with active UC were treated with GMA at our institute between June 2009 and September 2018. All patients received GMA therapy once or twice a week with the Adacolumn. Conventional medication was to be continued during the whole GMA treatment course. The clin-
\end{abstract}

ical response was retrospectively evaluated. Results: According to the partial Mayo score, remission was 33.3\%, significant efficacy $25.6 \%$, effective $25.6 \%$, and no response $15.4 \%$. The average partial Mayo score was $6.2 \pm 1.4$ at entry and significantly declined to $1.8 \pm 1.8$ after GMA sessions $(p<$ 0.0001 ). The average number of bowel movements was $9.5 \pm$ 5.6 at entry and significantly declined to $3.0 \pm 2.8$ after GMA sessions $(p<0.0001)$. In a comparison between the group treated with concomitant PSL and the group without PSL, the change in partial Mayo score or the number of bowel movements from entry to after GMA sessions was not significantly different. Among 24 patients treated by GMA with concomitant PSL, 75\% (18/24) became steroid free. Conclusions: The effect of GMA with concomitant PSL and that of GMA without PSL were not different, and GMA was effective irrespective of PSL administration. The present study showed that GMA had efficacy and led many UC patients treated by PSL to be steroid free with no safety concern in the real world, although there is the possibility of recruitment bias due to the retrospective nature of the study.

(c) 2020 The Author(s)

Published by S. Karger AG, Basel

\begin{tabular}{ll}
\hline KARGER & $\begin{array}{l}\text { ( 2020 The Author(s) } \\
\text { Published by S. Karger AG, Basel }\end{array}$ \\
E-Mail karger@karger.com & $\begin{array}{l}\text { This article is licensed under the Creative Commons Attribution- } \\
\text { NonCommercial-NoDerivatives 4.0 International License (CC BY- } \\
\text { NC-ND) (http://www.karger.com/Services/OpenAccessLicense). } \\
\text { Usage and distribution for commercial purposes as well as any dis- } \\
\text { tribution of modified material requires written permission. }\end{array}$
\end{tabular}

Keiji Matsuda, MD, PhD

Department of Surgery

Teikyo University School of Medicine

2-11-1 Kaga, Itabashi-ku, Tokyo 173-8605 (Japan)

E-Mail keiji@med.teikyo-u.ac.jp 


\section{Introduction}

The number of ulcerative colitis (UC) patients is increasing in Japan and other countries [1]. Steroids and immunosuppressants have been administered to UC patients. However, the chronic administration of these medications is associated with serious side effects such as treatment-related morbidity factors [2]. Selective depletion of myeloid lineage leucocytes by adsorptive granulocyte and monocyte apheresis (GMA) with an Adacolumn (JIMRO, Takasaki, Japan) was introduced as a nonpharmacologic treatment strategy in UC patients in 2000. GMA is aimed at reducing the elevated and activated leucocytes known to be sources of inflammatory cytokines [3]. GMA has been reported to be effective in clinical trials; however, the effect of concomitant prednisolone (PSL) has yet to be clarified.

In the present study we retrospectively examined the clinical data of patients who underwent GMA, and investigated the effect of concomitant PSL on GMA.

\section{Materials and Methods}

\section{Study Design and Objectives}

This retrospective study aimed at identifying disease background features. Thirty-nine patients with active UC were treated with GMA at our institute between June 2009 and September 2018. In Japan the indication for GMA is patients with moderate to severe UC, and GMA is officially approved by Japanese health insurance. The physicians in charge decided on GMA treatment and combination usage of PSL or other drugs. Our institute had no clear criteria for choosing GMA for UC patients. The retrospective exploration showed a kind of trend toward GMA being applied for moderate UC, steroid dependent and steroid refractory patients, and patients who worry about side effects of steroids.

\section{GMA Procedures}

All patients received GMA therapy once to twice a week with the Adacolumn as previously described [4]. Conventional medication was to be continued during the whole GMA treatment course.

\section{Evaluation of Efficacy}

During the GMA treatment period, patients' vital signs including heart rate, blood pressure, and respiration rate were monitored. Routine blood samples were taken at entry, after GMA treatment, and at regular intervals during the observation period. The primary efficacy measures included the partial Mayo score [5] and number of bowel movements. The included patients were assessed at entry and after treatment. A reduction of $90-100 \%$ in the partial Mayo score was defined as "remission"; 70-89\% decrease in symptoms was defined as "significant efficacy," 30-69\% as "effective," and $<30 \%$ as "no response" according to the previous report [6]. "Total efficacy" was the sum of remission, significant efficacy, and effective fractions.

Adsorptive GMA with and without Concomitant PSL
Table 1. Baseline demographic variables of the 39 patients with ulcerative colitis

\begin{tabular}{lc}
\hline Age, years & $37.9 \pm 19.5$ \\
Gender (male:female) & $21: 18$ \\
Medications (some overlapping) & \\
$\quad$ None & 2 \\
Prednisolone (systemic) & 25 \\
Prednisolone (enema) & 1 \\
5-ASA or sulphasalazine (oral) & 37 \\
5-ASA (enema) & 3 \\
Azathioprine (oral) & 8 \\
Tacrolimus (oral) & 4 \\
Infliximab & 1 \\
Observation period, months & $38 \pm 34$
\end{tabular}

Data are presented as $n$ or mean $\pm \mathrm{SD}$, as appropriate.

\section{Statistics}

When appropriate, numerical data are presented as mean \pm SD values. Comparison of demographic variables was done using either the Mann-Whitney U test or the Student $t$ test using the JMP statistical package (SAS Institute, Vary, NC, USA).

\section{Results}

\section{Baseline Demographic Variables}

Thirty-nine patients with active UC received GMA treatment sessions. The patients' main demographic variables are presented in Table 1. Systemic PSL was administered to 25 patients. Among the 39 patients included in this study, 4 did not complete the procedure for the following reasons: 2 allergic reactions (Grade 1, Common Terminology Criteria for Adverse Events Version 4.0), 1 patient's decision, and 1 difficulty of securing vascular access. No serious adverse event was observed during the GMA sessions. The frequency of GMA was as follows: once in 1 patient, 3 times in 2 patients, 5 in 1 patient, 7 in 2 patients, 9 in 1 patient, 10 in 11 patients, and 11 in 21 patients. The timing of evaluating the efficacy of GMA was 1-2 weeks after the last GMA session. Eleven cases comprised the steroid refractory group. Before the start of the first GMA session, the dose and timing of PSL in each case, respectively, was $20 \mathrm{mg}$ and 20 days, 30 and 5 days, 30 and 24 days, 30 and 30 days, 30 and 42 days, 40 and 3days, 50 and 7 days, 50 and 9 days, 50 and 10 days, 60 and 8 days, and 70 and 14 days. Five cases comprised the steroid dependent group. Before the start of the first GMA session, the duration of administration of PSL including a temporary withdrawal period in each case, re-

Inflamm Intest Dis 2020;5:36-41 
Table 2. Clinical efficacy outcomes after GMA sessions

\begin{tabular}{lr}
\hline Remission & $13(33.3)$ \\
Significant efficacy & $10(25.6)$ \\
Effective & $10(25.6)$ \\
No response & $6(15.4)$ \\
\hline
\end{tabular}

Data are presented as $n(\%)$.

spectively, was 1 year and 2 months, 1 year and 3 months, 4 years, 6 years, and 13 years.

\section{Clinical Efficacy Outcomes after GMA Sessions}

According to the partial Mayo score, remission was $33.3 \%$, significant efficacy $25.6 \%$, effective $25.6 \%$, and no response $15.4 \%$ (Table 2). Effective cases accounted for $84.6 \%$ of UC patients treated with GMA. Colonoscopic findings were classified according to the Mayo score [7], and those of patients who achieved mucosal healing are shown in Figures 1 and 2.

The clinical efficacy of GMA on changes in partial Mayo score evaluated at entry and after all GMA sessions (via line charts) is shown in Figure 1. The average partial Mayo score was $6.2 \pm 1.4$ at entry and significantly declined to $1.8 \pm 1.8$ after GMA sessions $(p<0.0001)$. Paired analyses for the partial Mayo scores at entry and after GMA were compared between the PSL (-) and PSL (+) groups in Figure 2. In both groups partial Mayo score was significantly decreased from entry to after GMA sessions. The average number of bowel movements was $9.5 \pm 5.6$ at entry and significantly declined to $3.0 \pm 2.8$ after GMA sessions $(p<0.0001)$.

\section{Effect of Concomitant PSL on GMA}

The partial Mayo score at entry of the GMA-treated group with/without concomitant PSL is shown in Figure 3. No significant difference was observed between the 2 groups. The change in partial Mayo score from entry to after GMA sessions of the GMA-treated group with/without concomitant PSL is shown in Figure 4. The change in score of the group without PSL was $3.6 \pm 2.8$, and that of the group with concomitant PSL $4.8 \pm 2.2$. There was no significant difference between them. Furthermore, the change in partial Mayo score from entry to after GMA sessions was studied among the steroid-naïve, dependent, and refractory groups. The average change, respectively, was $5.2,4.4$, and 4.5 . There was no significant difference among the 3 groups.

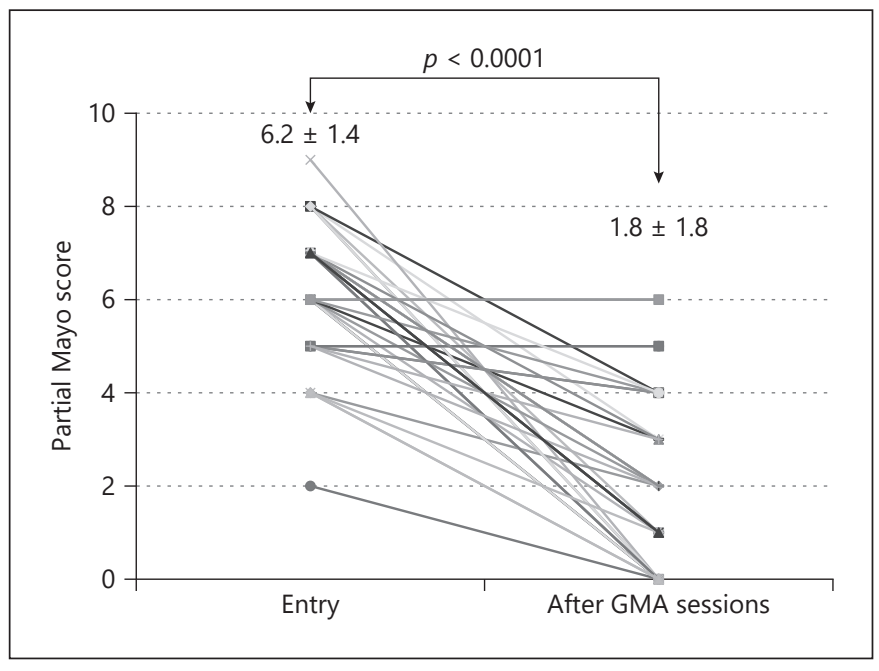

Fig. 1. The clinical efficacy of GMA on changes in partial Mayo score evaluated at entry and after all GMA sessions.

As for the number of bowel movements at entry of the GMA-treated group with/without concomitant PSL, no significant difference was observed between the 2 groups; and as for the change in the number of bowel movements from entry to after GMA sessions, there was no significant difference between them.

\section{Clinical Course of Patients Treated with GMA}

The 5-year cumulative nonoperative rate was $97.3 \%$. The clinical course of cases treated with GMA in combination with PSL is shown in Figure 5. Among the 24 patients treated with GMA with concomitant PSL, 75\% $(18 / 24)$ became steroid free.

\section{Discussion}

UC has a chronic nature, and patients need medications such as 5-ASA, thiopurines, PSL, or anti-TNF- $\alpha$ biologics throughout life. These might lead to drug dependency or loss of response, and adverse events often occur with conventional drugs. Nonpharmacological treatment like GMA would be suitable to avoid adverse side effects of drugs, especially corticosteroids. Suzuki et al. [4] showed efficacy of $85 \%$ and no severe adverse events in steroidnaïve UC patients. Fukunaga et al. [8] selected corticosteroid refractory patients with active UC and showed the efficacy of GMA in preventing UC relapse. Yokoyama et al. [9] compared steroid responder, dependent, and re-
38

Inflamm Intest Dis 2020;5:36-41 DOI: $10.1159 / 000505484$
Matsuda et al. 
Fig. 2. The paired analyses for the partial Mayo scores at entry and after GMA were compared between PSL (-) (a) and PSL (+) groups (b).

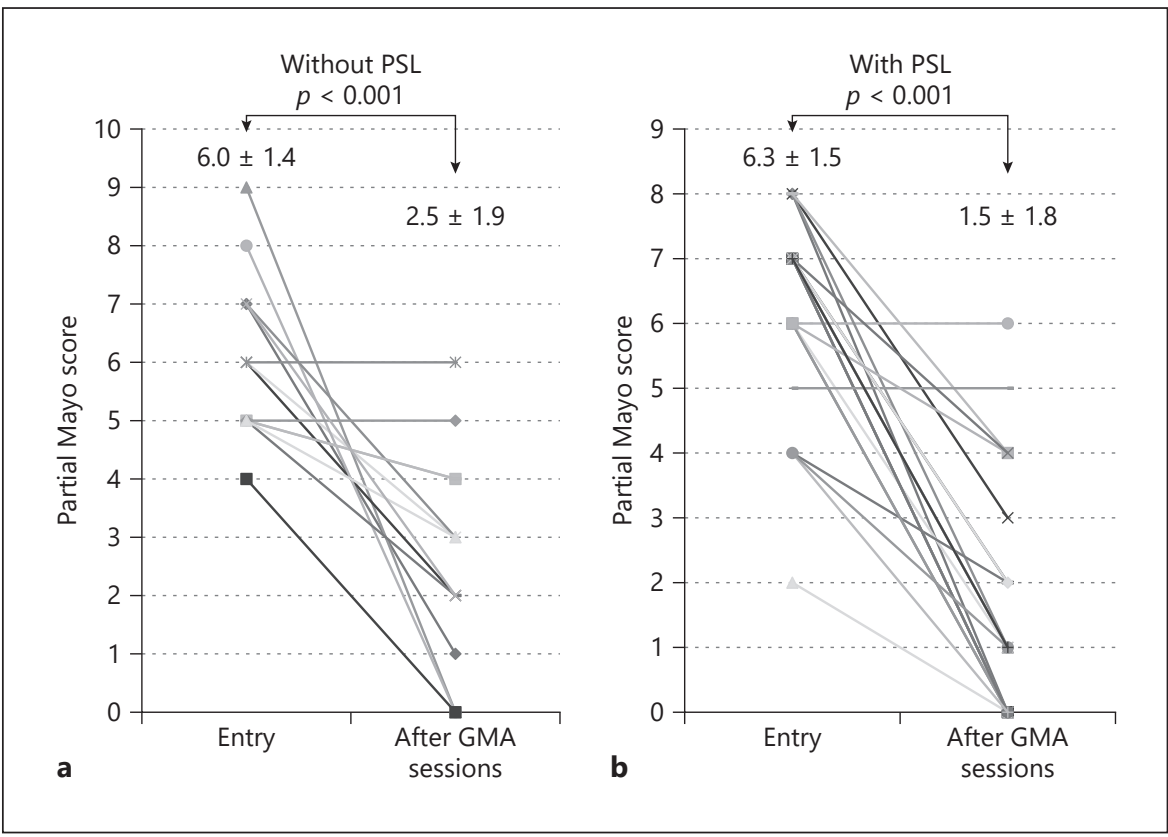

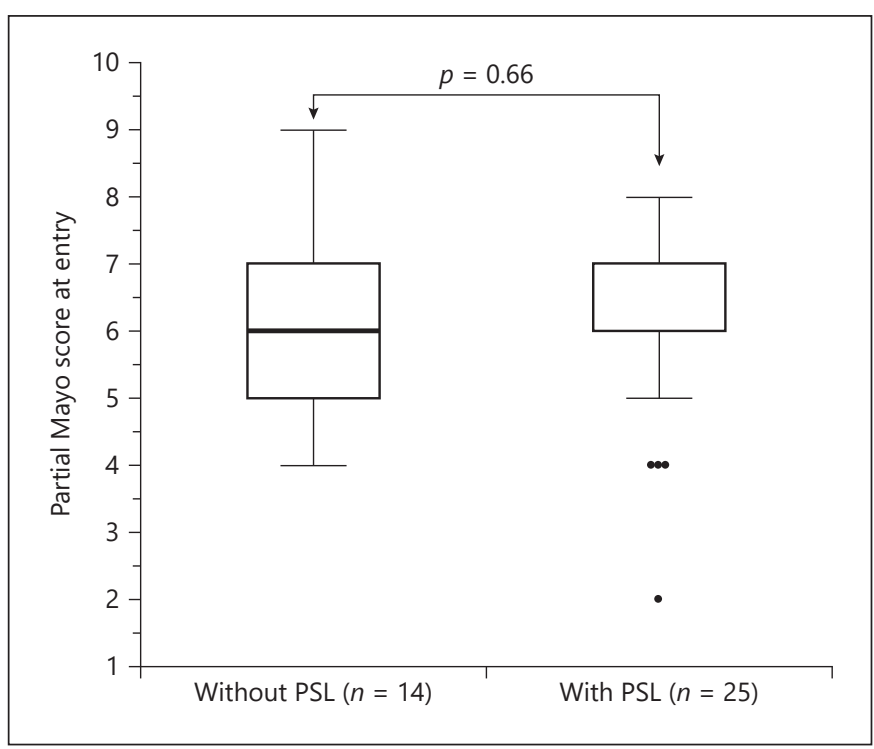

Fig. 3. Partial Mayo score at entry of GMA-treated group with/ without concomitant PSL.

fractory patients, and showed that steroid responder was a significant factor for maintaining remission. Although papers on GMA have been published, the comparison between GMA with PSL and GMA without PSL has never been studied. In the present study it is investigated.

The outcome of the present investigation might be summarized as follows. Thirty-nine patients received

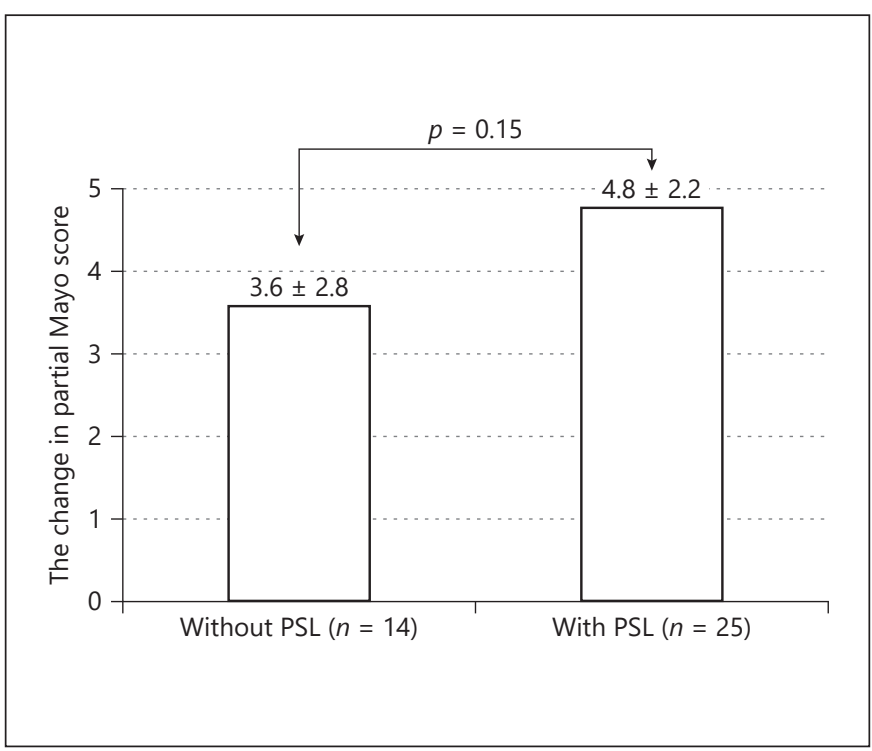

Fig. 4. The change in partial Mayo score from entry to after GMA sessions of GMA-treated group with/without concomitant PSL.

GMA sessions for an active UC were retrospectively reviewed. All 39 patients had active disease in spite of receiving conventional medications (5-ASA or PSL). Remission was achieved in $33.3 \%$, significant efficacy in $25.6 \%$, effective in $25.6 \%$, and no response in $15.4 \%$. The total efficacy rate was $84.6 \%$, which was close to the report of Suzuki et al. [4], and the $15.4 \%$ no response rate was 


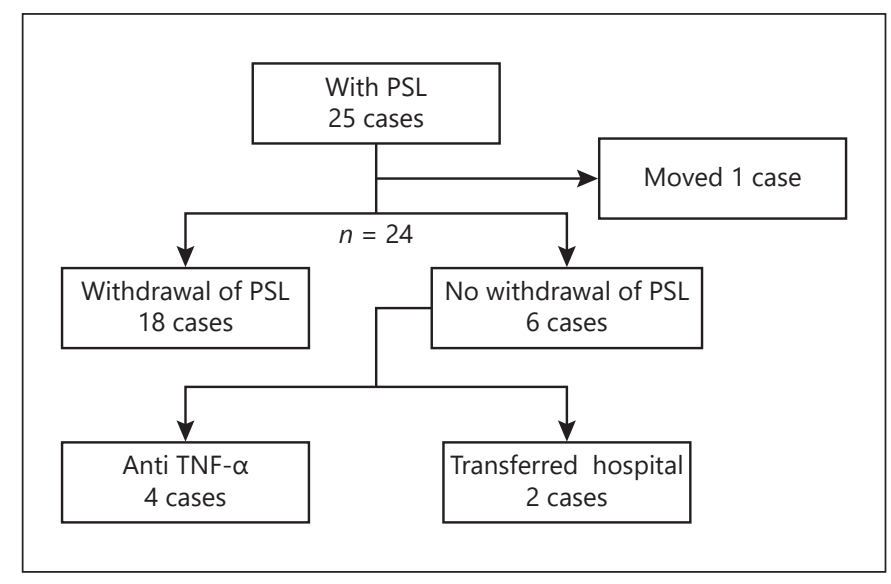

Fig. 5. The clinical course of cases treated by GMA in combination with PSL.

similar to $18.0 \%$ in the report of Yokoyama et al. [9]. The efficacy of GMA with PSL was not significantly different from that of GMA without PSL.

A significant difference was observed in the change in partial Mayo score and number of bowel movements between entry and after GMA sessions. The cumulative nonoperative rate was $98.4 \%$, and $75 \%$ of patients became steroid free. Since this report was a clinical result and the patients' careers were varied, it was impossible to identify patients with effective GMA. Furthermore, there is the possibility of recruitment bias due to the retrospective nature of the study. In 39 patients, only 5 comprised the steroid dependent group, and their steroid-free remission was only $60 \%$. The small number of patients in this group might have influenced the high steroid-free rate. As this is a retrospective study, the effects of other treatments could not be eliminated. However, regardless of the combined use of corticosteroid, there was a good response rate to GMA.

Since GMA has fewer side effects than conventional drugs and has a high response rate, it is considered a suitable treatment method to try at an early stage of UC [4,
$10,11]$. Whether PSL is administered or not, GMA should be a choice of treatment for patients with active UC.

\section{Acknowledgments}

We are thankful to all the professionals working in the renal center in Teikyo University Hospital for their assistance in performing GMA.

\section{Statement of Ethics}

In Japan GMA with the Adacolumn is an officially approved therapeutic option for patients with active UC. Additionally, informed consent was obtained from all patients after explaining GMA to them. Further, adherence was made to the Principle of Good Clinical Practice and the Helsinki Declaration at all times. This retrospective exploration of data from diagnostic and therapy at our hospital was approved by the Institutional Review Board (Ethics Committee) of the Teikyo University (16-032) on August 23, 2016. This Ethics Committee approval is comprehensive and not specific to the present study.

\section{Disclosure Statement}

The authors declare no conflict of interest.

\section{Funding Sources}

There are no funding sources.

\section{Author Contributions}

K.M. treated the patients, gathered the patient data, and wrote the manuscript. K.O., Y.O., T.Yag, M.T., Y.F., A.H., R.S., T.O., T.H., T.T., J.T., H.I., K.N., H.A., A.I., K.A., S.K., T.Yam, Y.K., Y.T., and Y.H. contributed to the daily medical treatment of the cases. Y.S. and F.K. were responsible for histopathological diagnosis. Y.H. represented our surgical department and supervised the writing of the manuscript. All authors significantly contributed to this study and approved the final manuscript.

\section{References}

1 Ng SC, Shi HY, Hamidi N, Underwood FE, Tang W, Benchimol EI, et al. Worldwide incidence and prevalence of inflammatory bowel disease in the 21st century: a systematic review of population-based studies. Lancet. 2018 Dec;390(10114):2769-78.

2 Tominaga K, Nakano M, Hoshino M, Kanke K, Hiraishi H. Efficacy, safety and cost analy- ses in ulcerative colitis patients undergoing granulocyte and monocyte adsorption or receiving prednisolone. BMC Gastroenterol. 2013 Mar;13(1):41.

3 Saniabadi AR, Tanaka T, Ohmori T, Sawada $\mathrm{K}$, Yamamoto T, Hanai H. Treating inflammatory bowel disease by adsorptive leucocytapheresis: a desire to treat without drugs.
World J Gastroenterol. 2014 Aug;20(29): 9699-715.

4 Suzuki Y, Yoshimura N, Saniabadi AR, Saito Y. Selective granulocyte and monocyte adsorptive apheresis as a first-line treatment for steroid naïve patients with active ulcerative colitis: a prospective uncontrolled study. Dig Dis Sci. 2004 Apr;49(4):565-71.
40

Inflamm Intest Dis 2020;5:36-41 DOI: $10.1159 / 000505484$
Matsuda et al. 
5 Lewis JD, Chuai S, Nessel L, Lichtenstein GR, Aberra FN, Ellenberg JH. Use of the noninvasive components of the Mayo score to assess clinical response in ulcerative colitis. Inflamm Bowel Dis. 2008 Dec;14(12):1660-6.

6 Lai YM, Yao WY, He Y, Jiang X, Gu YB, Chen $\mathrm{MH}$, et al. Adsorptive granulocyte and monocyte apheresis in the treatment of ulcerative colitis: the first multicenter study in China. Gut Liver. 2017 Mar;11(2):216-25.

7 Paine ER. Colonoscopic evaluation in ulcerative colitis. Gastroenterol Rep (Oxf). 2014 Aug;2(3):161-8.
8 Fukunaga K, Yokoyama Y, Kamokozuru K, Nagase K, Nakamura S, Miwa H, et al. Adsorptive granulocyte/monocyte apheresis for the maintenance of remission in patients with ulcerative colitis: a prospective randomized, double blind, sham-controlled clinical trial. Gut Liver. 2012 Oct;6(4):427-33.

9 Yokoyama Y, Watanabe K, Ito H, Nishishita M, Sawada K, Okuyama Y, et al. Factors associated with treatment outcome, and longterm prognosis of patients with ulcerative colitis undergoing selective depletion of myeloid lineage leucocytes: a prospective multicenter study. Cytotherapy. 2015 May;17(5): 680-8.
10 Suzuki Y, Yoshimura N, Fukuda K, Shirai K Saito Y, Saniabadi AR. A retrospective search for predictors of clinical response to selective granulocyte and monocyte apheresis in patients with ulcerative colitis. Dig Dis Sci. 2006 Nov;51(11):2031-8.

11 Tanaka T, Sugiyama S, Goishi H, Kajihara T, Akagi M, Miura T. Treatment of children and adolescents with ulcerative colitis by adsorptive depletion of myeloid lineage leucocytes as monotherapy or in combination with low dose prednisolone after failure of first-line medications. BMC Gastroenterol. 2013 Aug; 13(1):130. 